\title{
Estrutura de assembléias de Muscidae (Diptera) no Paraná: uma análise por modelos nulos ${ }^{1}$
}

\author{
Jaime Iván Rodríguez-Fernández², Claudio José Barros de Carvalho² \& Mauricio Oswaldo Moura ${ }^{3}$
}

${ }^{1}$ Contribuição número 1587 do Departamento de Zoologia, Universidade Federal do Paraná.

${ }^{2}$ Departamento de Zoologia, Universidade Federal do Paraná. Caixa Postal 19020, 81531-980 Curitiba-PR, Brasil. formycusub@yahoo.com.br, cjbcarva@ufpr.br

${ }^{3}$ Departamento de Ciências Biológicas, Centro de Ciências Agrárias e Ambientais. Caixa Postal 3010, 85010-990 Guarapuava-PR moura@unicentro.br

\begin{abstract}
Muscidae (Diptera) assemblage structure in Paraná: a null model analysis. Community structure is often thought to be determined by interspecific competition. Critics of this idea state that we must first demonstrate structure with null models to test whether structure indeed exists. Here, we use 179 predatory and saprophagic species of flies in the family Muscidae (Diptera) that were captured in Malaise traps in six locations in Paraná State during one year of study. To test community structure, we generated five presence-absence (1-0) matrices: two by trophic guilds, two by habitat, and one general matrix (taxonomic). Two indices of co-occurrence (C) and covariance (V) of species were calculated in the matrices developed through 5,000 Monte Carlo randomizations. Randomizations followed two different assumptions: 1) fixed number of species per location, and 2) constant proportions of species at all locations. Comparisons with null-model communities showed that "taxonomic" species assemblage had a false structure, while "ecological" species assemblages had true structure. Although the ecological assemblages are consistent with the theory of interspecific competition as a cause of community structure, it remains possible that other causes of structure may also be important.
\end{abstract}

KEYWORDS. Randomization; species co-ocurrence; coexistence; competition; community ecology.

\begin{abstract}
RESUMO. Estrutura de assembléias de Muscidae (Diptera) no Paraná: uma análise por modelos nulos. Geralmente pensase que a estrutura das comunidades está determinada pela competição interespecífica. Os críticos desta idéia indicam que devemos primeiramente demonstrar a estrutura com modelos nulos par testar se a estrutura realmente existe. Aqui, utilizamos 179 espécies predadoras e saprófagas de moscas da família Muscidae (Diptera) que foram capturadas com armadilha Malaise em seis locais no Estado do Paraná, durante um ano de estudo. Para testar a estrutura das comunidades, geramos cinco matrizes de presença-ausência (1-0): duas por guildas tróficas, duas por tipo de habitat e uma matriz geral (taxonômica). Dois índices de co-ocorrência $(\mathrm{C})$ e covariância $(\mathrm{V})$ de espécies foram calculados nas matrizes desenvolvidas através de 5000 aleatorizações de Monte Carlo. Estas seguiram duas diferentes premissas: 1) número de espécies por local fixo, e 2) proporções constantes de espécies em todos os locais. Comparações com modelos nulos de comunidades mostram que a assembléia "taxonômica" de espécies tem uma falsa estrutura, enquanto assembléias de espécies "ecológicas" têm uma estrutura verdadeira. Enquanto as assembléias ecológicas são consistentes com a teoria de competição interespecífica como uma causa da estrutura das comunidades, é possível que outras causas possam também ser importantes.
\end{abstract}

PALAVRAS CHAVE. Aleatorização; co-ocorrência de espécies; coexistência; competição; ecologia de comunidades.

A composição de espécies dentro das comunidades ecológicas pode ser explicada por distintos processos, como por exemplo: regras de união (Diamond 1975), estrutura de tamanhos (Hutchinson 1959), proporcionalidade de guildas (Mikkelson 1993; Wilson 1989), estados favorecidos (Fox 1987) e subconjuntos hierárquicos (Patterson 1987). A escola clássica (macarthuriana) em ecologia de comunidades estabelecia que a competição interespecífica era a principal força que determinava a estrutura das comunidades (Cody \& Diamond 1975). Teoricamente, " $n$ " espécies não podem coexistir em menos de que " $n$ " nichos, já que a exclusão competitiva (Gause 1934) impediria a coexistência de espécies com necessidades ecológicas idênticas. Ao final da década de 70, Daniel Simberloff e seus associados estudaram os dados originais de vários trabalhos da escola clássica, questionando muitas das conclusões desses pesquisadores (Connor \& Simberloff 1979; Gotelli \& Graves 1996) e, propondo uma nova forma de testar o efeito da competição. A crítica fundamental de Simberloff e colaboradores aos estudos anteriores, foi a de que os padrões descritos poderiam ser obtidos por diferentes mecanismos, dificultando a avaliação crítica da teoria (Arita \& Rodriguez 2002).

Ao fazer uma análise da existência e da magnitude dos efeitos da competição interespecífica em comunidades animais, Schoener (1983) e Connell (1983) encontraram uma ampla gama de comunidades que seriam estruturadas por competição. No entanto, ao fazer uma Meta-Análise testando a validade das regras de reunião de Diamond (1975), Gotelli \& McCabe (2002) mostraram que grupos ectotérmicos (exceto formigas) têm um padrão mais aleatório (não estruturado) do que grupos endotérmicos. No caso de artrópodes, alguns exemplos de competição interespecífica relacionada com a estrutura das comunidades incluem: hierarquias etológicas de dominância (Punttila et al. 1996), relações de dominância (Andersen 1992), agressão interespecífica (Cole 1983), interações químicas (Andersen et al. 1991), territorialidade (Fox et al. 1985), 
mosaicos espaciais (Ryti \& Case 1992), interações de competição-colonização (Holway 1999) e deslocamento competitivo (Porter \& Savignano 1990).

Muscidae é uma linhagem com mais de 4500 espécies descritas com habitats variados (Carvalho et al. 2005); os grupos funcionais, segundo o estágio ontogenético e/ou a espécie variam desde predadores facultativos ou obrigatórios de outros invertebrados, saprófagos, polinófagos, fitófagos, endoparasitos e hematófagos (Skidmore 1985; Carvalho et al. 2005). Em regiões de alta latitude e altitude formam uma alta proporção da fauna (Pont 1986).

Dada a amplitude de guildas tróficas de Muscidae, a escassez de trabalhos analisando a estrutura de comunidades naturais de dípteros (e.g. Gilbert et al. 1985; Grillet \& Barrera 1997; Chaves \& Añez 2004) e evidências de que em grandes escalas as comunidades de Muscidae não variam muito (Costacurta et al. 2003) este trabalho tem como objetivo principal determinar se existe uma estruturação regional nas comunidades de Muscidae do Paraná. Especificamente, será testada a relação entre estrutura e diversidade biológica tanto em nível de toda comunidade quanto de guildas.

\section{MATERIALE MÉTODOS}

Os espécimes utilizados foram coletados entre 1987-1988, dentro do PROFAUPAR (Projeto de Levantamento da Fauna Entomológica no Estado do Paraná - Marinoni \& Dutra 1991) em Antonina, Fênix e Jundiaí do Sul. Marinoni \& Dutra (1991) mostraram que essas três localidades formam um conjunto separado das demais localidades no primeiro ano de coleta, pois são as que apresentam a maior temperatura máxima anual e a maior temperatura mínima anual, além de manter-se como um agrupamento característico na análise geral de todas as variáveis metereológicas.

Hatschbach apud Marinoni \& Dutra (1991) também dividiu os locais do PROFAUPAR em dois agrupamentos: agrupamento $\mathrm{A}$ com alto poder de recuperação (Antonina, Fênix, Jundiaí do Sul e Guarapuava) e agrupamento B, com poder de recuperação menor. O poder de recuperação representaria empiricamente a capacidade de cada local de restabelecer a vegetação original, após distúrbio ocasionado pela extração de árvores nas últimas décadas.

Para incrementar a representatividade espacial, taxonômica e ecológica, também foram usados os dados de outras três localidades (Colombo, Ponta Grossa e Guarapuava) amostradas no primeiro ano do PROFAUPAR, 1986-1987 (Costacurta et al. 2003).

Detalhes dos procedimentos de amostragem e características dos locais foram indicados por Marinoni \& Dutra (1991).

Estrutura das comunidades. Para a análise da estrutura das comunidades, os dados foram organizados como matrizes de presença-ausência. Cada linha da matriz representa uma espécie e cada coluna representa uma localidade. A entrada na matriz representa a presença (1) ou ausência (0) da espécie na localidade. Foram construídas cinco matrizes: uma para o conjunto total das espécies das seis localidades, uma para a guilda trófica que agrupa as espécies predadoras, uma para a guilda trófica que agrupa as espécies saprófagas, uma para as espécies das localidades analisadas por Costacurta et al. (2003), e uma só para as espécies do conjunto de espécies das localidades deste trabalho. Todas estas matrizes foram analisadas segundo a metodologia sugerida por Gotelli (2000).

O hábito alimentar das espécies foi inferido a partir dos dados da morfologia, taxonomia e filogenia, segundo Carvalho (2002).

Escolha dos índices. Vários índices foram descritos para resumir os padrões de co-ocorrência de espécies (Schluter 1984; Peres-Neto et al. 2001). Gotelli (2000) comparou o desempenho de quatro índices de co-ocorrência e nove diferentes algoritmos de modelos nulos em relação a erros estatísticos do tipo I e tipo II. Nesse estudo observou-se que o índice C ou "C score" de Stone \& Roberts (1990) e o índice V ou "V ratio" de Schluter (1984) não eram propensos a erros tipo I e II e podiam detectar padrões significativos, mesmo em conjuntos de dados com ruído.

$\mathrm{O}$ índice de co-ocorrência da matriz (Índice C) tem bom poder para detectar pares de espécies que não co-ocorrem freqüentemente, enquanto que a razão de variância (Índice V) revela padrões não aleatórios nos totais das linhas e colunas da matriz. Por essas razões, esses dois índices (C e V) foram escolhidos.

Escolha do modelo nulo. Três alternativas são possíveis para utilizar os totais das linhas ou colunas das matrizes nos modelos nulos: mantendo as somatórias fixas, proporcionais ou equiprováveis. Deste modo, seria possível construir $3^{2}=9$ modelos nulos. Apesar das nove possibilidades serem logicamente plausíveis, Gotelli (2000) demonstrou que modelos com a somatória de linhas fixas possuem pouca probabilidade do erro tipo I. Portanto, só foram utilizados os modelos nos quais a somatória das linhas é fixa (F), podendo a somatória das colunas ser fixa $(\mathrm{F})$, proporcional $(\mathrm{P})$ ou equiprovável $(\mathrm{E})$.

Linha fixa versus coluna fixa (FxF): Neste algoritmo ambas as somatórias de linhas e colunas da matriz original são mantidas constantes; portanto diferenças nas freqüências de ocorrência de cada espécie (somatória de linhas) e diferenças no número de espécies por local (somatória de colunas) são preservadas.

Linha fixa versus coluna proporcional (FxP): Neste algoritmo, a distribuição das espécies é proporcional ao total observado nas colunas. Isto permite aos totais das colunas variarem, como no modelo equiprovável; porém, pode refletir diferenças entre sítios como no modelo fixo.

Um outro modelo normalmente usado é o de linha fixa versus colunas euqiprováveis (FxE). Neste modelo as comunidades são consideradas como equivalentes. Como em nosso estudo, existem comunidades claramente distintas, este algorítmo não foi utilizado.

Para cada uma das cinco análises, calculamos os dois índices (Índice C e Índice V) com a combinação dos dois algoritmos previamente mencionados. Todas as análises foram feitas com o programa ECOSIM (Gotelli \& Entsminger 2003). 


\section{RESULTADOS}

Todas as subfamílias neotropicais de Muscidae foram representadas no material amostrado que compreende 179 espécies nominais. Neste conjunto, também se encontraram representantes das três guildas tróficas registradas para os adultos de Muscidae: predadores, saprófagos e hematófagos (Tabela I).

As análises de modelos nulos com os diferentes índices (Índice C, Índice V) e diferentes algoritmos (fixo versus fixo e fixo versus proporcional), para os distintos cenários (por localidades, por guildas tróficas e o conjunto geral) mostrados na Tabela II, sugerem distintos resultados. Distingue-se que ambos os índices, o Índice $\mathrm{C}$ e o Índice $\mathrm{V}$, têm valores significativamente menores nas matrizes originais por localidade, ou seja, as menos estruturadas são as matrizes por localidade e progressivamente se fazem mais estruturadas até a matriz geral, tudo em função da significância: quanto maior o valor do Índice V, maior a covariância entre totais por sítio e totais e, portanto, existe maior variação de espécies por sítio. Um maior valor médio do Índice $\mathrm{C}$ mostra menor co-ocorrência de pares de espécies por sítio e, portanto, assembléias mais estruturadas. Já, entre as matrizes por localidade a regra também parece ser de assembléias não estruturadas.

A única matriz que tem todos os valores calculados (entre os dois índices e os diferentes algoritmos), demonstrando que não se detecta algum tipo de estrutura, é a matriz com localidades do agrupamento B (locais com menor poder de recuperação).

Nas análises da matriz de localidades tipo A (locais com maior poder de recuperação), para o índice $\mathrm{C}$, os algoritmos mostram estruturas ao acaso. Com o Índice $\mathrm{V}$, o algoritmo fixo versus proporcional mostra um padrão estruturado.

Nas matrizes das guildas, a regra parece ser de assembléias estruturadas. Para predadores com o Índice C, ambos os algoritmos mostram um padrão estruturado. Já com o Índice V, o algoritmo Fixo versus proporcional mostra-se ao acaso. Para os saprófagos, todos os valores do índice $\mathrm{C}$ mostram assembléias estruturadas. Apenas o Índice V, com o algoritmo fixo versus proporcional, mostra ausência de estrutura.

Os mesmos resultados de todas as alternativas entre os índices e os algoritmos encontrados para não predadores, são observados para os dados agrupados numa matriz geral.

Com o Índice V, só quando mantemos as colunas proporcionais, obtemos um valor que indica uma assembléia estruturada em uma análise: a análise do agrupamento A.

Uma primeira interpretação dos resultados da análise de estrutura sugere, pelos valores da matriz geral, que as assembléias de imagos de Muscidae são altamente estruturadas. A separação por guildas tróficas poderia confirmar a observação inicial, na matriz geral, sobre o alto grau de estruturação das assembléias de imagos de Muscidae.

Nas matrizes por localidade é observado que as localidades do agrupamento B tem muito poucas espécies exclusivas por local (entre o 5 a $11 \%$ ), ou seja, $90 \%$ ou mais é compartilhado (diversidade beta alta). Uma diversidade beta alta também significa que na matriz, a maioria das células das três localidades mostra a mesma espécie presente. Por outro lado, as demais localidades do agrupamento A possuem entre 22 a $66 \%$ de espécies exclusivas, ou seja, uma diversidade beta muito menor (pouca similaridade de espécies).

\section{DISCUSSÃO}

Generalizando que a primeira causa (não a única) que determina a estrutura das comunidades é a competição interespecífica, pode-se afirmar que processos locais (competição interespecífica) estão determinando padrões de distribuição de espécies em uma escala maior (Schluter \& Ricklefs 1993).

Ao restringir o alvo de estudo numa guilda trófica, estamos descartando as espécies que não coexistem no mesmo nível trófico e, portanto, não tem sobreposição de requerimento de recursos (Simberloff \& Dayan 1991).

Toda a discussão anterior mostraria como a segregação entre as espécies determina a estrutura das assembléias de Muscidae. Porém, os dados iniciais podem mostrar que não é a "segregação" mas a "agregação" entre espécies (Gotelli \& Rohde 2002) que está determinando os padrões observados.

Se a segregação observada (possivelmente produto da competição interespecífica) é o fator que determina a estrutura, no momento que agrupamos a matriz de predadores com a matriz de não predadores, estamos analisando um pool de espécies com várias interações espúrias. Assim, muitos pares consistiram de espécies com diferentes usos de recursos (não interagindo diretamente), o que poderia provocar um efeito de diluição, como proposto por Diamond \& Gilpin (1982). Nesta perspectiva, após agrupar as matrizes de predadores e não predadores, a análise da matriz geral resultante deveria mostrar padrões mais coerentes com a hipótese de assembléias não estruturadas. Ao observar os resultados, vemos que não é o caso e, além disso, os índices mostram alguns valores superiores aos índices das matrizes de guildas, contrariamente ao esperado.

$\mathrm{O}$ alto valor da diversidade beta nas localidades do agrupamento B gera uma matriz de presença-ausência com a seguinte tendência:

$\begin{array}{lll}1 & 1 & 1 \\ 1 & 1 & 1 \\ 1 & 1 & 1\end{array}$

Mais o efeito de uma matriz desse tipo, combinada com outra matriz de outras três localidades com uma diversidade beta muito menor, poderia produzir uma matriz com a seguinte tendência:

$\begin{array}{llllll}1 & 1 & 1 & 0 & 0 & 0 \\ 1 & 1 & 1 & 0 & 0 & 0 \\ 1 & 1 & 1 & 0 & 0 & 0 \\ 0 & 0 & 0 & 1 & 1 & 1 \\ 0 & 0 & 0 & 1 & 1 & 1 \\ 0 & 0 & 0 & 1 & 1 & 1\end{array}$


Tabela I. Espécies de Muscidae de seis localidades do Estado do Paraná: Antonina=AN, Fênix=FE, e Jundiaí do Sul=JU, Colombo=CO, Ponta Grossa=PG e Guarapuava= GU. CO, PG e GU durante o período de agosto de 1986 a julho de 1987 e AN, FE e JU durante agosto de 1987 a julho de 1988. Hábito trófico: saprófago (s), predador (p) ou hematófago (h) de cada espécie. Presença=1, ausência $=0$. As entradas de célula com o valor (1) indicam presença da espécie no local ao passo que sua ausência é indicada por (0).

\begin{tabular}{|c|c|c|c|c|c|c|c|}
\hline Espécies & AN & FE & $\mathrm{JU}$ & $\mathrm{CO}$ & PG & GU & Hábito \\
\hline Atherigona orientalis Schiner, 1868 & 0 & 1 & 1 & 0 & 0 & 0 & $\mathrm{~s}$ \\
\hline Bithoracochaeta calopus (Bigot, 1885) & 1 & 1 & 1 & 1 & 1 & 1 & $\mathrm{p}$ \\
\hline Bithoracochaeta flavicoxa Malloch, 1934 & 1 & 0 & 1 & 0 & 0 & 0 & $\mathrm{p}$ \\
\hline Bithoracochaeta leucoprocta (Wiedemann, 1830) & 0 & 0 & 0 & 0 & 1 & 0 & $\mathrm{p}$ \\
\hline Bithoracochaeta plumata Albuquerque, 1955 & 0 & 1 & 0 & 0 & 0 & 1 & $\mathrm{p}$ \\
\hline Bithoracochaeta sp. 1 & 0 & 0 & 1 & 0 & 0 & 0 & $\mathrm{p}$ \\
\hline Bithoracochaeta sp. 2 & 0 & 0 & 1 & 0 & 0 & 0 & $\mathrm{p}$ \\
\hline Bithoracochaeta sp. 3 & 1 & 0 & 0 & 0 & 0 & 0 & $\mathrm{p}$ \\
\hline Bithoracochaeta sp. 4 & 1 & 0 & 0 & 0 & 0 & 0 & $\mathrm{p}$ \\
\hline Bithoracochaeta sp. 5 & 0 & 1 & 0 & 0 & 0 & 0 & $\mathrm{p}$ \\
\hline Brontaea debilis (Williston, 1896) & 0 & 1 & 0 & 0 & 0 & 0 & $\mathrm{~s}$ \\
\hline Byopyrellia bipuncta (Wiedemann, 1830) & 1 & 1 & 1 & 0 & 0 & 0 & $\mathrm{~s}$ \\
\hline Cariocamyia maculosa Snyder,1951 & 0 & 0 & 0 & 0 & 0 & 1 & $\mathrm{~s}$ \\
\hline Chaetagenia stigmatica Malloch, 1928 & 1 & 0 & 0 & 0 & 0 & 0 & $\mathrm{~s}$ \\
\hline Coenosia sp.1 & 0 & 0 & 0 & 1 & 0 & 0 & $\mathrm{p}$ \\
\hline Coenosia sp.2 & 0 & 0 & 0 & 1 & 1 & 1 & $\mathrm{p}$ \\
\hline Coenosia sp.3 & 0 & 0 & 0 & 0 & 1 & 0 & $\mathrm{p}$ \\
\hline Coenosia sp.4 & 0 & 0 & 0 & 0 & 1 & 0 & $\mathrm{p}$ \\
\hline Coenosia sp. 5 & 0 & 1 & 1 & 0 & 0 & 0 & $\mathrm{p}$ \\
\hline Coenosia sp. 6 & 0 & 1 & 1 & 0 & 0 & 0 & $\mathrm{p}$ \\
\hline Coenosia sp. 7 & 0 & 0 & 1 & 0 & 0 & 0 & $\mathrm{p}$ \\
\hline Cordiluroides listrata Albuquerque, 1954 & 1 & 0 & 1 & 0 & 0 & 0 & $\mathrm{p}$ \\
\hline Cordiluroides megalopyga Albuquerque, 1954 & 1 & 1 & 1 & 0 & 0 & 1 & $\mathrm{p}$ \\
\hline Cordiluroides sp. 1 & 0 & 0 & 1 & 0 & 0 & 0 & $\mathrm{p}$ \\
\hline Cordiluroides sp. $n .1$ & 0 & 0 & 1 & 0 & 0 & 0 & $\mathrm{p}$ \\
\hline Cyrtoneurina alifusca Couri, 1982 & 0 & 0 & 1 & 0 & 0 & 0 & $\mathrm{~s}$ \\
\hline Cyrtoneurina biseta Snyder, 1954 & 1 & 0 & 0 & 0 & 0 & 0 & $\mathrm{~s}$ \\
\hline Cyrtoneurina sp. 1 & 0 & 0 & 1 & 0 & 0 & 0 & $\mathrm{~s}$ \\
\hline Cyrtoneuropsis fuscicosta (Curran, 1934) & 0 & 0 & 1 & 0 & 0 & 0 & $\mathrm{~s}$ \\
\hline Cyrtoneuropsis incognita (Snyder, 1954) & 0 & 0 & 0 & 1 & 1 & 1 & $\mathrm{~s}$ \\
\hline Cyrtoneuropsis maculipennis (Macquart, 1843) & 1 & 1 & 1 & 0 & 0 & 0 & $\mathrm{~s}$ \\
\hline Cyrtoneuropsis mellina (Stein, 1918) & 0 & 0 & 0 & 1 & 1 & 1 & $\mathrm{~s}$ \\
\hline Cyrtoneuropsis mimica (Snyder, 1954) & 1 & 0 & 0 & 0 & 0 & 0 & $\mathrm{~s}$ \\
\hline Cyrtoneuropsis pararescita (Couri, 1995) & 0 & 0 & 1 & 0 & 0 & 0 & $\mathrm{~s}$ \\
\hline Cyrtoneuropsis polystigma (Wulp, 1896) & 0 & 0 & 1 & 0 & 0 & 0 & $\mathrm{~s}$ \\
\hline Cyrtoneuropsis rescita (Walker, 1861) & 0 & 0 & 1 & 0 & 0 & 0 & $\mathrm{~s}$ \\
\hline Cyrtoneuropsis sp. 1 & 0 & 0 & 1 & 0 & 0 & 0 & $\mathrm{~s}$ \\
\hline Cyrtoneuropsis sp. 2 & 0 & 0 & 0 & 1 & 1 & 1 & $\mathrm{~s}$ \\
\hline Cyrtoneuropsis veniseta (Stein, 1904) & 1 & 0 & 0 & 0 & 0 & 0 & $\mathrm{~s}$ \\
\hline Dolichophaonia catharinensis (Carvalho, 1983) & 0 & 0 & 0 & 1 & 1 & 1 & $\mathrm{~s}$ \\
\hline Dolichophaonia elongata (Albuquerque, 1958) & 1 & 0 & 1 & 0 & 0 & 0 & $\mathrm{~s}$ \\
\hline Dolichophaonia giacomeli (Carvalho, 1981) & 0 & 0 & 0 & 0 & 0 & 1 & $\mathrm{~s}$ \\
\hline Dolichophaonia plaumanni (Carvalho, 1983) & 1 & 0 & 1 & 1 & 1 & 1 & $\mathrm{~s}$ \\
\hline Dolichophaonia santoamarensis (Albuquerque, 1958) & 0 & 0 & 1 & 0 & 0 & 1 & $\mathrm{~s}$ \\
\hline Dolichophaonia sp.1 & 0 & 0 & 0 & 1 & 0 & 1 & $\mathrm{~s}$ \\
\hline Dolichophaonia sp.2 & 0 & 0 & 0 & 0 & 0 & 1 & $\mathrm{~s}$ \\
\hline Dolichophaonia sp.3 & 0 & 0 & 0 & 0 & 0 & 1 & $\mathrm{~s}$ \\
\hline Dolichophaonia sp. $n .1$ & 0 & 0 & 1 & 0 & 0 & 0 & $\mathrm{~s}$ \\
\hline Dolichophaonia sp. n. 2 & 0 & 0 & 1 & 0 & 0 & 0 & $\mathrm{~s}$ \\
\hline Gênero novo $1 s p . n .1$ (Azeliinae, Reinwardtiini) & 1 & 1 & 1 & 0 & 0 & 0 & $\mathrm{~s}$ \\
\hline Gênero novo 1 sp. $n .1$ (Coenosiinae, Coenosiini) & 0 & 0 & 1 & 0 & 0 & 0 & $\mathrm{p}$ \\
\hline Gênero novo 2 sp.n. 2 (Azeliinae, Reinwardtiini) & 0 & 1 & 1 & 0 & 0 & 0 & $\mathrm{~s}$ \\
\hline Gênero Novo A, espécie 1 & 0 & 0 & 0 & 0 & 1 & 1 & $\mathrm{~s}$ \\
\hline Gênero Novo B, espécie 1 & 0 & 0 & 0 & 1 & 1 & 1 & $\mathrm{~s}$ \\
\hline Gênero Novo C, espécie 1 & 0 & 0 & 0 & 0 & 0 & 1 & $\mathrm{~s}$ \\
\hline Helina praecipua (Walker, 1853) & 1 & 1 & 1 & 1 & 1 & 1 & $\mathrm{~s}$ \\
\hline Helina sp. 1 & 0 & 0 & 0 & 0 & 1 & 1 & \\
\hline
\end{tabular}


Tabela I. Continuação

\begin{tabular}{|c|c|c|c|c|c|c|c|}
\hline Espécies & $\mathrm{AN}$ & $\mathrm{FE}$ & $\mathrm{JU}$ & $\mathrm{CO}$ & $\mathrm{PG}$ & $\mathrm{GU}$ & Hábito \\
\hline Helina sp. 2 & 0 & 0 & 0 & 1 & 1 & 1 & $\mathrm{~s}$ \\
\hline Helina sp. 3 & 0 & 0 & 0 & 0 & 1 & 1 & $\mathrm{~s}$ \\
\hline Helina sp. 4 & 0 & 0 & 0 & 1 & 0 & 0 & $\mathrm{~s}$ \\
\hline Helina sp. 5 & 1 & 0 & 0 & 0 & 0 & 0 & $\mathrm{~s}$ \\
\hline Helina sp. 6 & 0 & 1 & 0 & 0 & 0 & 0 & $\mathrm{~s}$ \\
\hline Helina sp. 7 & 0 & 1 & 0 & 0 & 0 & 0 & $\mathrm{~s}$ \\
\hline Helina sp. 8 & 0 & 0 & 1 & 0 & 0 & 0 & $\mathrm{~s}$ \\
\hline Helina sp. 9 & 0 & 0 & 1 & 0 & 0 & 0 & $\mathrm{~s}$ \\
\hline Helina sp. 10 & 0 & 0 & 1 & 0 & 0 & 0 & $\mathrm{~s}$ \\
\hline Hemichlora scordalus (Walker, 1861) & 1 & 0 & 0 & 0 & 0 & 0 & $\mathrm{~s}$ \\
\hline Limnophora sp. 1 & 0 & 1 & 1 & 0 & 0 & 0 & $\mathrm{p}$ \\
\hline Limnophora $\mathrm{sp} .2$ & 0 & 0 & 1 & 0 & 0 & 0 & $\mathrm{p}$ \\
\hline Limnophora sp. 3 & 1 & 0 & 1 & 0 & 0 & 0 & $\mathrm{p}$ \\
\hline Limnophora sp. 4 & 1 & 0 & 1 & 0 & 0 & 0 & $\mathrm{p}$ \\
\hline Lispe sp. 1 & 1 & 0 & 0 & 0 & 0 & 0 & $\mathrm{p}$ \\
\hline Lispe sp. 2 & 1 & 0 & 0 & 0 & 0 & 0 & $\mathrm{p}$ \\
\hline Micropotamia sp. 1 & 1 & 0 & 0 & 0 & 0 & 0 & $\mathrm{~s}$ \\
\hline Morellia dendropanacis Pamplona \& Couri, 1995 & 0 & 1 & 1 & 1 & 1 & 1 & $\mathrm{~s}$ \\
\hline Morellia humeralis (Stein,1918) & 0 & 1 & 0 & 0 & 1 & 1 & $\mathrm{~s}$ \\
\hline Morellia maculipennis (Macquart, 1846) & 0 & 0 & 0 & 0 & 1 & 1 & $\mathrm{~s}$ \\
\hline Morellia paulistensis Pamplona \& Mendes, 1995 & 0 & 1 & 0 & 0 & 0 & 0 & $\mathrm{~s}$ \\
\hline Morellia violacea (Robineau-Desvoidy,1830) & 1 & 1 & 1 & 1 & 1 & 1 & $\mathrm{~s}$ \\
\hline Morellia xanthoptera Pamplona, 1986 & 1 & 1 & 1 & 0 & 1 & 0 & $\mathrm{~s}$ \\
\hline Mydaea plaumanni Snyder, 1941 & 1 & 1 & 1 & 1 & 1 & 1 & $\mathrm{~s}$ \\
\hline Mydaea sp. 1 & 1 & 0 & 0 & 0 & 0 & 0 & $\mathrm{~s}$ \\
\hline Mydaea sp. 2 & 0 & 1 & 1 & 0 & 0 & 0 & $\mathrm{~s}$ \\
\hline Mydaea sp. 3 & 0 & 0 & 1 & 0 & 0 & 0 & $\mathrm{~s}$ \\
\hline Mydaea sp. 4 & 0 & 0 & 1 & 0 & 0 & 0 & $\mathrm{~s}$ \\
\hline Myospila meditabunda (Fabricius, 1781) & 0 & 0 & 1 & 0 & 0 & 0 & $\mathrm{~s}$ \\
\hline Myospila obscura (Shannon \& Del Ponte, 1926) & 1 & 0 & 1 & 1 & 1 & 1 & $\mathrm{~s}$ \\
\hline Neivamyia flavicornis (Malloch, 1928) & 1 & 0 & 0 & 0 & 0 & 0 & $\mathrm{~h}$ \\
\hline Neodexiopsis barviventris Couri \& Albuquerque, 1979 & 0 & 0 & 1 & 0 & 0 & 0 & $\mathrm{p}$ \\
\hline Neodexiopsis elegans Couri \& Albuquerque, 1979 & 0 & 0 & 1 & 0 & 0 & 0 & $\mathrm{p}$ \\
\hline Neodexiopsis emmesa (Malloch, 1934) & 0 & 0 & 0 & 1 & 1 & 1 & $\mathrm{p}$ \\
\hline Neodexiopsis flavipalpis Albuquerque, 1956 & 1 & 1 & 1 & 1 & 1 & 1 & $\mathrm{p}$ \\
\hline Neodexiopsis fulvifrontis Couri \& Albuquerque, 1979 & 0 & 0 & 1 & 0 & 0 & 0 & $\mathrm{p}$ \\
\hline Neodexiopsis grupo ovatta $\mathrm{sp} .1$ & 0 & 0 & 1 & 0 & 0 & 0 & $\mathrm{p}$ \\
\hline Neodexiopsis grupo ovatta sp. $n .1$ & 1 & 1 & 0 & 0 & 0 & 0 & $\mathrm{p}$ \\
\hline Neodexiopsis grupo ovatta sp. $n .2$ & 1 & 0 & 0 & 0 & 0 & 0 & $\mathrm{p}$ \\
\hline Neodexiopsis grupo ovatta sp. $n .3$ & 0 & 0 & 1 & 0 & 0 & 0 & $\mathrm{p}$ \\
\hline Neodexiopsis neoaustralis Snyder, 1957 & 0 & 0 & 1 & 1 & 1 & 1 & $\mathrm{p}$ \\
\hline Neodexiopsis nigerrima (Malloch, 1934) & 1 & 0 & 1 & 1 & 1 & 1 & $\mathrm{p}$ \\
\hline Neodexiopsis rustica Albuquerque, 1956 & 0 & 1 & 1 & 0 & 0 & 0 & $\mathrm{p}$ \\
\hline Neodexiopsis setipuncta Snyder, 1957 & 0 & 0 & 0 & 1 & 1 & 1 & $\mathrm{p}$ \\
\hline Neodexiopsis sp. 1 & 0 & 0 & 0 & 1 & 1 & 1 & $\mathrm{p}$ \\
\hline Neodexiopsis sp.2 & 0 & 0 & 0 & 0 & 1 & 1 & $\mathrm{p}$ \\
\hline Neodexiopsis sp. 3 & 1 & 0 & 0 & 1 & 1 & 1 & $\mathrm{p}$ \\
\hline Neodexiopsis sp. 4 & 0 & 0 & 0 & 1 & 1 & 1 & $\mathrm{p}$ \\
\hline Neodexiopsis sp. 5 & 0 & 0 & 1 & 1 & 1 & 1 & $\mathrm{p}$ \\
\hline Neodexiopsis sp. 6 & 1 & 0 & 0 & 1 & 1 & 1 & $\mathrm{p}$ \\
\hline Neodexiopsis sp. 7 & 0 & 0 & 0 & 1 & 0 & 1 & $\mathrm{p}$ \\
\hline Neodexiopsis sp. 8 & 0 & 0 & 0 & 1 & 0 & 0 & $\mathrm{p}$ \\
\hline Neodexiopsis sp. 9 & 0 & 0 & 1 & 1 & 1 & 1 & $\mathrm{p}$ \\
\hline Neodexiopsis sp.10 & 0 & 0 & 0 & 1 & 1 & 1 & $\mathrm{p}$ \\
\hline Neodexiopsis sp. 11 & 1 & 0 & 1 & 0 & 0 & 0 & $\mathrm{p}$ \\
\hline Neodexiopsis sp. 12 & 1 & 0 & 0 & 0 & 0 & 0 & $\mathrm{p}$ \\
\hline Neodexiopsis sp. 13 & 1 & 0 & 1 & 0 & 0 & 0 & $\mathrm{p}$ \\
\hline Neodexiopsis sp. 14 & 1 & 0 & 0 & 0 & 0 & 0 & $\mathrm{p}$ \\
\hline Neodexiopsis sp. 15 & 1 & 0 & 0 & 0 & 0 & 0 & $\mathrm{p}$ \\
\hline Neodexiopsis sp. 16 & 0 & 0 & 1 & 0 & 0 & 0 & $\mathrm{p}$ \\
\hline Neodexiopsis vulgaris Couri \& Albuquerque, 1979 & 0 & 0 & 0 & 1 & 1 & 1 & $\mathrm{p}$ \\
\hline
\end{tabular}


Tabela I. Continuação

\begin{tabular}{|c|c|c|c|c|c|c|c|}
\hline Espécies & $\mathrm{AN}$ & $\mathrm{FE}$ & JU & $\mathrm{CO}$ & PG & GU & Hábito \\
\hline Neomuscina atincticosta Snyder, 1949 & 0 & 0 & 0 & 1 & 1 & 1 & $\mathrm{~s}$ \\
\hline Neomuscina capalta Snyder, 1949 & 0 & 0 & 1 & 0 & 0 & 0 & $\mathrm{~s}$ \\
\hline Neomuscina currani Snyder, 1949 & 1 & 1 & 0 & 0 & 0 & 0 & $\mathrm{~s}$ \\
\hline Neomuscina goianensis Lopes \& Khouri, 1995 & 0 & 0 & 1 & 0 & 0 & 0 & $\mathrm{~s}$ \\
\hline Neomuscina inflexa (Stein, 1918) & 1 & 1 & 1 & 1 & 1 & 1 & $\mathrm{~s}$ \\
\hline Neomuscina instabilis Snyder, 1949 & 0 & 1 & 0 & 0 & 0 & 0 & $\mathrm{~s}$ \\
\hline Neomuscina neosimilis Snyder, 1942 & 0 & 1 & 1 & 0 & 0 & 0 & $\mathrm{~s}$ \\
\hline Neomuscina nudistigma Snyder, 1949 & 0 & 0 & 1 & 0 & 0 & 0 & $\mathrm{~s}$ \\
\hline Neomuscina pictipennis pictipennis (Bigot, 1878) & 0 & 1 & 1 & 1 & 1 & 0 & $\mathrm{~s}$ \\
\hline Neomuscina sanespra Snyder, 1949 & 1 & 0 & 0 & 0 & 0 & 0 & $\mathrm{~s}$ \\
\hline Neomuscina schadei Snyder, 1949 & 1 & 1 & 1 & 1 & 1 & 1 & $\mathrm{~s}$ \\
\hline Neomuscina sp. 5 & 1 & 0 & 0 & 0 & 0 & 0 & $\mathrm{~s}$ \\
\hline Neomuscina sp. 1 & 0 & 0 & 0 & 1 & 1 & 0 & $\mathrm{~s}$ \\
\hline Neomuscina sp. 2 & 0 & 0 & 0 & 1 & 1 & 1 & $\mathrm{~s}$ \\
\hline Neomuscina sp. 3 & 0 & 0 & 0 & 1 & 0 & 0 & $\mathrm{~s}$ \\
\hline Neomuscina sp. 4 & 0 & 0 & 0 & 1 & 1 & 0 & $\mathrm{~s}$ \\
\hline Neomuscina tinctinervis (Stein, 1918) & 0 & 0 & 0 & 1 & 1 & 1 & $\mathrm{~s}$ \\
\hline Neomuscina zosteris (Shannon \& Del Ponte, 1926) & 0 & 0 & 1 & 1 & 1 & 1 & $\mathrm{~s}$ \\
\hline Neurotrixa sp. 1 & 0 & 0 & 0 & 1 & 1 & 1 & $\mathrm{~s}$ \\
\hline Neurotrixa sp. 2 & 0 & 0 & 0 & 1 & 1 & 1 & $\mathrm{~s}$ \\
\hline Phaonia advena Snyder, 1957 & 0 & 0 & 0 & 1 & 1 & 1 & $\mathrm{~s}$ \\
\hline Phaonia annulata (Albuquerque, 1957) & 0 & 0 & 0 & 1 & 1 & 1 & $\mathrm{~s}$ \\
\hline Phaonia bigoti (Albuquerque, 1957) & 0 & 0 & 0 & 1 & 1 & 0 & $\mathrm{~s}$ \\
\hline Phaonia grajauensis (Albuquerque, 1957) & 0 & 0 & 0 & 1 & 1 & 1 & $\mathrm{~s}$ \\
\hline Phaonia lentiginosa Snyder, 1957 & 0 & 0 & 0 & 1 & 1 & 1 & $\mathrm{~s}$ \\
\hline Phaonia nigriventris (Albuquerque, 1954) & 0 & 0 & 1 & 1 & 1 & 1 & $\mathrm{~s}$ \\
\hline Phaonia praesuturalis (Stein, 1904) & 0 & 0 & 0 & 0 & 1 & 0 & $\mathrm{~s}$ \\
\hline Phaonia shannoni Carvalho \& Pont, 1993 & 0 & 0 & 0 & 1 & 1 & 1 & $\mathrm{~s}$ \\
\hline Phaonia similata (Albuquerque, 1957) & 0 & 0 & 1 & 0 & 0 & 0 & $\mathrm{~s}$ \\
\hline Phaonia sp.1 & 0 & 0 & 0 & 1 & 1 & 1 & $\mathrm{~s}$ \\
\hline Phaonia sp. 2 & 0 & 0 & 0 & 1 & 1 & 1 & $\mathrm{~s}$ \\
\hline Phaonia sp.3 & 0 & 0 & 0 & 1 & 1 & 1 & $\mathrm{~s}$ \\
\hline Phaonia sp.4 & 0 & 0 & 0 & 0 & 1 & 1 & $\mathrm{~s}$ \\
\hline Phaonia sp.5 & 0 & 0 & 0 & 0 & 1 & 1 & $\mathrm{~s}$ \\
\hline Phaonia trispila (Bigot, 1885) & 0 & 0 & 0 & 1 & 1 & 1 & $\mathrm{~s}$ \\
\hline Philornis masoni Couri, 1986 & 0 & 0 & 0 & 0 & 1 & 0 & $\mathrm{~s}$ \\
\hline Philornis sp. 1 & 0 & 0 & 1 & 0 & 0 & 0 & $\mathrm{~s}$ \\
\hline Philornis sp. 2 & 0 & 0 & 1 & 0 & 0 & 0 & $\mathrm{~s}$ \\
\hline Pilispina pilitibia Albuquerque, 1954 & 0 & 0 & 0 & 1 & 1 & 1 & $\mathrm{p}$ \\
\hline Plumispina similis Costacurta \& Carvalho, 2003 & 0 & 1 & 1 & 1 & 1 & 1 & $\mathrm{p}$ \\
\hline Plumispina sp. $n .1$ & 0 & 0 & 1 & 0 & 0 & 0 & $\mathrm{p}$ \\
\hline Plumispina sp. $n .2$ & 1 & 0 & 0 & 0 & 0 & 0 & $\mathrm{p}$ \\
\hline Polietina bicolor Albuquerque, 1956 & 0 & 0 & 1 & 0 & 1 & 1 & $\mathrm{~s}$ \\
\hline Polietina minor Albuquerque, 1956 & 1 & 1 & 1 & 0 & 0 & 0 & $\mathrm{~s}$ \\
\hline Polietina nigra Couri \& Carvalho, 1996 & 1 & 0 & 0 & 0 & 0 & 0 & $\mathrm{~s}$ \\
\hline Polietina orbitalis (Stein, 1904) & 1 & 1 & 1 & 1 & 1 & 1 & $\mathrm{~s}$ \\
\hline Polietina prima (Couri \& Machado, 1990) & 1 & 0 & 0 & 0 & 0 & 0 & $\mathrm{~s}$ \\
\hline Polietina steini (Enderlein, 1927) & 1 & 1 & 0 & 0 & 1 & 0 & $\mathrm{~s}$ \\
\hline Polietina univittata Couri \& Carvalho, 1995 & 0 & 0 & 0 & 1 & 1 & 1 & $\mathrm{~s}$ \\
\hline Pseudoptilolepis fulvapoda Snyder, 1949 & 0 & 0 & 1 & 1 & 1 & 1 & $\mathrm{~s}$ \\
\hline Psilochaeta pampiana (Shannon \& Del Ponte, 1926) & 0 & 0 & 1 & 1 & 1 & 0 & $\mathrm{~s}$ \\
\hline Sarcopromusca pruna (Shannon \& del Ponte, 1926) & 0 & 0 & 1 & 0 & 0 & 0 & $\mathrm{~s}$ \\
\hline Souzalopesmyia sp. n. 1 & 1 & 0 & 0 & 0 & 0 & 0 & $\mathrm{~s}$ \\
\hline Stomopogon argentina (Snyder, 1957) & 0 & 0 & 1 & 0 & 0 & 0 & $\mathrm{p}$ \\
\hline Stomopogon hirtitibia (Stein, 1911) & 1 & 0 & 0 & 0 & 0 & 0 & $\mathrm{p}$ \\
\hline Stomopogon sp. 1 & 0 & 0 & 0 & 1 & 1 & 0 & $\mathrm{p}$ \\
\hline Stomopogon sp. 2 & 0 & 0 & 0 & 0 & 1 & 0 & $\mathrm{p}$ \\
\hline Xenomorellia sp. 1 & 0 & 0 & 0 & 1 & 1 & 1 & $\mathrm{p}$ \\
\hline Xenomorellia sp. 2 & 0 & 0 & 0 & 0 & 1 & 0 & $\mathrm{~s}$ \\
\hline Xenomorellia sp. 3 & 0 & 0 & 0 & 1 & 1 & 0 & $\mathrm{~s}$ \\
\hline Xenothoracochaeta sp. 1 & 0 & 0 & 1 & 0 & 0 & 0 & $\mathrm{~s}$ \\
\hline
\end{tabular}


Tabela II. Análises de modelos nulos para assembléias de espécies de Muscidae em seis localidades do Estado do Paraná com dois índices de coocorrência e dois algoritmos diferentes para a construção de modelos nulos. Agrupamento A: corresponde a Antonina, Fênix e Jundiaí (locais com temperatura média máxima e mínima anual maior poder de recuperação maior - 1987-1988). Agrupamento B: corresponde a Colombo, Ponta Grossa e Guarapuava (1986-1987). Valores em negrito representam as assembléias estruturadas. P = valor de significância médio calculado após 5000 aleatorizações do modelo. Obs $=$ valor estimado para a matriz real. Calc $=$ valor médio calculado pelo modelo. FxF= linhas fixas versus colunas fixas, $\mathrm{FxP}=$ linhas fixas versus colunas proporcional.

\begin{tabular}{|c|c|c|c|c|c|c|c|c|c|c|c|}
\hline & & \multirow{2}{*}{\multicolumn{2}{|c|}{ Geral }} & \multicolumn{4}{|c|}{ Guildas } & \multicolumn{4}{|c|}{ Locais } \\
\hline & & & & \multicolumn{2}{|c|}{ Saprófagos } & \multicolumn{2}{|c|}{ Predadores } & \multicolumn{2}{|c|}{ Agrupamento A } & \multicolumn{2}{|c|}{ Agrupamento B } \\
\hline & & Índice $\mathrm{C}$ & Índice $\mathrm{V}$ & Índice $\mathrm{C}$ & Índice $\mathrm{V}$ & Índice C & Índice $\mathrm{V}$ & Índice C & Índice $\mathrm{V}$ & Índice $\mathrm{C}$ & Índice V \\
\hline & Obs & 1,46 & 6,97 & 1,49 & 4,44 & 1,43 & 4,00 & 0,45 & 0,45 & 0,12 & 1,32 \\
\hline \multirow[t]{2}{*}{$\mathrm{FxF}$} & Calc & 1,28 & - & 1,32 & - & 1,23 & - & 0,44 & - & 0,12 & - \\
\hline & $\mathrm{P}$ & $<\mathbf{0 , 0 1}$ & - & $<\mathbf{0 , 0 1}$ & - & $<\mathbf{0 , 0 1}$ & - & 0,12 & - & 0,33 & - \\
\hline \multirow[t]{2}{*}{ FxP } & Calc & 1,46 & 6,97 & 1,34 & 3,33 & 1,29 & 2,78 & 0,46 & 0,44 & 0,12 & 1,06 \\
\hline & $\mathrm{P}$ & $<0,01$ & 0,09 & $\mathbf{0 , 0 1}$ & 0,20 & $<0,01$ & 0,17 & 0,94 & 0,04 & 0,70 & 0,33 \\
\hline
\end{tabular}

Esta última é uma matriz altamente estruturada, pois representa a ausência da metade das espécies em oposição da presença da outra metade de espécies. Em nosso caso, essa estrutura deve surgir pela agregação de indivíduos nos dois tipos de locais e os altos valores da diversidade beta nos locais do agrupamento B. Uma matriz hipotética com a mesma estrutura é apresentada por Gotelli (2000), para analisar o efeito de uma matriz altamente estruturada.

Por outro lado, a detecção do padrão estruturado das guildas tróficas não parece ser um artifício, mas um padrão real. De maneira geral, os valores do "Índice V", nas guildas tróficas indicam um padrão não estruturado, mais este índice se baseia nas covariâncias dos totais das colunas e das linhas, e não na estrutura interna da matriz (Schluter 1984). Portanto, para localidades com uma diversidade beta elevada (locais com muitas espécies em comum), as covariâncias positivas tenderiam a ser maiores (assembléias não estruturadas), porém na estrutura interna da matriz existe um padrão estruturado.

Desta forma, o Índice $\mathrm{V}$ pode estar detectando agregação de espécies e não segregação (ao menos com o algoritmo Fixo versus Proporcional), e o Índice C mostra o padrão estruturado para as guildas de espécies de Muscidae.

Uma possível razão a ser futuramente testada é que as assembléias estão saturadas de espécies, ou seja, fatores locais (por exemplo, tamanhos mínimos de populações viáveis) e não regionais seriam os determinantes da composição de espécies de Muscidae (Srivastava 1999). Competição aparente (compartilhamento de inimigos naturais) (Morris et al. 2004), ou fatores evolutivos como a estrutura filogenética das comunidades (Webb et al. 2002) poderiam também produzir esse padrão.

Agradecimentos. Ao Dr. Claudio José von Zuben (Universidade Estadual Paulista, Campus de Rio Claro) e Dr. Sionei Ricardo Bonatto (Universidade Federal do Paraná) pelas sugestões na versão inicial do manuscrito. Aos avaliadores anônimos pelas sugestões e, à Coordenação de Aperfeiçoamento de Pessoal de Nível Superior (CAPES) pela bolsa de Mestrado ao autor sênior. CJBC é grato ao Conselho Nacional de Desenvolvimento Científico e Tecnológico (CNPq) pela bolsa de produtividade em pesquisa (processo 304.148/ 2002-4).

\section{REFERÊNCIAS}

Andersen, A. N. 1992. Regulation of "momentary" diversity by dominant species in exceptionally rich ant communities of the seasonal tropics. American Naturalist 140: 401-420.

Andersen, A. N.; M. S. Blum \& T. H. Jones. 1991. Venom alkaloids in Monomorium "rothsteini" Forel repel other ants: is this the secret to success by Monomorium in Australian ant communities? Oecologia 88: 157-160.

Arita, H. T. \& P. Rodríguez. 2002. Ecología geográfica y macroecología. P. 63-80. In: J. Llorente \& J. J. Morrone (Eds.). Introducción a la biogeografía en Latinoamérica: Teorías, conceptos, métodos y aplicaciones. Las prensas de Ciencias, Facultad de Ciencias Puras, México, $277 \mathrm{p}$.

Cadotte, M. W. \& T. Fukami. 2005. Dispersal, spatial scale, and species diversity in a hierarchically structured experimental landscape. Ecology Letters 8: 548-557.

Brown, W. L., Jr. \& E. O. Wilson. 1956. Character displacement. Systematic Zoology 5: 49-64.

Carvalho; C. J. B. de (Ed.). 2002. Muscidae (Diptera) of the Neotropical Region: Taxonomy. Curitiba. Editora Universidade Federal do Paraná. 287 p.

Carvalho, C. J. B. de; M. S. Couri; A. C. Pont; D. M. Pamplona \& S. M. Lopes. 2005. A Catalogue of the Muscidae (Diptera) of the Neotropical Region. Zootaxa 860: 1-282.

Chaves, L. F. \& N. Añez. 2004. Species co-occurrence and feeding behavior in sand fly transmission of American cutaneous leishmaniasis in western Venezuela. Acta Tropica 92: 219-24.

Cody, M. L. \& J. M. Diamond 1975. Ecology and Evolution of communities. Cambridge, Harvard Univ. Press, 212 p.

Connell, J. 1983. On the prevalence and relative importance of interspecific competition: evidence from field experiments. American Naturalist 122: 661-696.

Connor, E. F. \& D. S. Simberloff. 1979. The assembly of species communities: chance or competition? Ecology 60: 1132-1140.

Costacurta, N. C.; R. C. Marinoni, \& C. J. B. de Carvalho. 2003. Fauna de Muscidae (Díptera) em três localidades do Estado de Paraná, capturada com armadilha Malaise. Revista Brasileira de Entomologia 47: 389-397.

Cole, B. J. 1983. Assembly of mangrove ant communities: patterns of geographical distribution. Journal of Animal Ecology 52: 339-347.

Diamond, J. M. \& M. E. Gilpin. 1982. Examination of the "null" model of Connor and Simberloff for species co-occurrences on islands. Oecologia 52: 64-74.

Fox, B. J. 1987. Species assembly and the evolution of community structure. Evolutionary Ecology 1: 201-213.

Fox B. J.; M. D. Fox \& E. Archer. 1985. Experimental confirmation of competition between two dominant species of Iridomyrmex (Hymenoptera: Formicidae). Australian Journal of Ecology 10: $105-110$. 
Gause, G. F. 1934. The struggle for existence. Baltimore, Williams and Wilkin, $193 \mathrm{p}$.

Gilbert, F. S.; Harding, E. F.; Line, J. M. \& Perry, H. 1985. Morphological approaches to community structure in hoverflies (Diptera, Syrphidae). Proceedings of the Royal Society of London B: $115-130$

Gotelli, N. J. 2000. Null model analysis of species co-ocurrence patterns. Ecology 81: 2606-2621.

Gotelli, N. J. and G. L. Entsminger. 2006. EcoSim: Null models software for ecology. Version 7. Acquired Intelligence Inc. \& Kesey-Bear. Jericho, VT 05465. Disponível em: <http:// homepages.together.net/ gentsmin/ecosim.htm>. [acessado em 15 de Outubro de 2003].

Gotelli, N. J. \& D. J. McCabe. 2002. Species co-occurrence: a metaanalysis of J. M. Diamond's assembly rules model. Ecology 83: 2091-2096.

Gotelli, N. J. \& G. R. Graves. 1996. Null models in ecology. Smithsonian Inst. Press, 368 p.

Gotelli, N. J. \& K. Rohde. 2002. Co-occurrence of ectoparasites of marine fishes: null model analysis. Ecology Letters 5: 86-94

Grillet, M. E. \& R. Barrera. 1997. Spatial and temporal abundance, substrate partitioning and species co-occurrence in a guild of Neotropical blackflies (Diptera: Simuliidae). Hydrobiologia 345: 197-208.

Holway, D. 1999. Competitive mechanisms underlying the displacement of native ants by the invasive argentine ant. Ecology 80: 238251.

Marinoni, R. C. \& R. R. C. Dutra. 1991. Levantamento da fauna entomológica no estado de Paraná. Introdução Situações climática e florística de oito pontos de coleta. Dados faunísticos de agosto de 1986 a julho de 1987. Revista Brasileira de Zoologia 8: 31-73.

Mikkelson, G. M. 1993. How do food webs fall apart - a study of changes in trophic structure during relaxation on habitat fragments. Oikos 67: 539-547.

Morris, R. J.; O. T. Lewis \& H. C. J. Godfray. 2004. Experimental evidence for apparent competition in a tropical forest food web. Nature 428: 310-313.

Patterson, B. D. 1987. The principle of nested subsets and its implications for biological conservation. Conservation Biology 1: $323-334$.
Peres-Neto, P. R.; J. D. Olden \& D. A. Jackson. 2001. Environmentally constrained null models: site suitability as occupancy criterion. Oikos 93: 110-120

Pont, A. C. 1986. Family Muscidae. p. 57-214. In: A. S. Sóos \& L. Papp (ed.), Catalog of the Paleartic Diptera. Vol. 11. Hungarian Natural History Museum, Budapest, 311 p.

Porter, S. D. \& D. A. Savignano. 1990. Invasion of polygene fire ants decimates native ants and disrupts arthropod community. Ecology 71: 2095-2106

Punttila, P.; Y. Haila \& H. Tukia. 1996. Ant communities in taiga clearcuts: habitat effects and species interactions. Ecography 19: $16-28$.

Ryti, R. T. \& T. J. Case. 1992. The role of neighborhood competition in the spacing and diversity of ant communities. American Naturalist 139: 355-374.

Schluter, D. 1984. A variance test for detecting species associations, with some applications. Ecology 65: 998-1005.

Schluter, D. \& R. E. Ricklefs. 1993. Community diversity: an introduction to the problem. p. 1-10. In: R. E. Ricklefs \& D. Schluter (Eds.): Species Diversity: Historical and Geographical Perspectives. Chicago, University of Chicago Press, 414 p.

Schoener, T. W. 1983. Field experiments on interespecific competition. American Naturalist 122: 240-285.

Simberloff, D. \& T. -Dayan. -1991. The guild concept and the structure of ecological communities. Annual Review of Ecology and Systematics 22: 115-43.

Skidmore, P. 1985. The biology of the Muscidae of the World. Lancaster, Junk Publishers, 550 p.

Srivastava, D. S. 1999. Using local-regional richness plots to test for species saturation: Pitfalls and potentials. Journal of Animal Ecology 68: 1-17.

Stone, L. \& A. Roberts. 1990. The checkerboard score and species distributions. Oecologia 85: 74-79.

Webb, C. O.; D. D. Ackerly; M. A. McPeek \& M. J. Donoghue. 2002. Phylogenies and community ecology. Annual Review of Ecology and Systematic 33: 475-505.

Wilson, J. B. 1989. A null model of guild proportionality, applied to stratification of a New Zealand temperate rain forest. Oecologia 80: $263-267$. 